SCIENTIFIC LETTER

\title{
Replacement of extracted permanent pacemaker or defibrillator leads by cannulation of veins using the femoral "drag through" technique
}

\section{R J Schilling}

$\mathrm{T}$ he development of specialised equipment has allowed permanent pacemaker lead extraction using minimally invasive techniques, ${ }^{12}$ but despite this the major complication rate is approximately $1-3 \% .{ }^{12}$ One reason for this is that the fragile subclavian vein, superior vena cava, and right atrium may tear during the process of cutting away the fibrous tissue that enfolds the leads in these regions. Drawing the body of the lead, which has the narrowest profile (cf. the tip and electrodes), through this fibrous sleeve from below, using a femoral approach, avoids the need for this dissection. Furthermore, the dissection required when using a superior approach to expose the subclavian vein can be painful and time consuming. When using a femoral approach only superficial dissection to free the leads and remove the suture sleeves is required and therefore the procedure can be performed under local anaesthetic. The major disadvantage of the femoral approach is that it does not provide access for a replacement lead. This study investigated the feasibility of a modification to conventional femoral lead extraction, providing venous access for introduction of a new pacing/ implantable cardioverter-defibrillator (ICD) lead along the channel occupied by the extracted lead.

\section{METHODS \\ Patients}

Any patients requiring lead extraction and immediate replacement of an ipsilateral pacing/ICD system were considered suitable for lead replacement using a "drag through" technique. Patients considered unsuitable were those in whom there was evidence of lead infection, or crushing of the lead between the clavicle and the first rib, reflecting a suboptimal implantation route.

\section{Lead extraction procedure}

The procedure was performed under local anaesthetic (with two exceptions, see table 1) with additional analgesia at the patient's request. Venography was performed on the ipsilateral side and a temporary pacing wire was inserted via the left femoral vein. The generator was removed, the lead(s) requiring extraction cut, freed to the retaining sleeves/sutures, and the sutures removed. A 16 French sheath was inserted in the right femoral vein and either a basket and deflecting wire (Byrd femoral workstation) or a needle-eye-snare were introduced (Cook UK, Ltd). A normal stylet was advanced into the pacing/ICD lead before being snared in order to preserve the lumen. As soon as the lead was grasped this stylet was removed.

The drag through technique was then performed by cutting the pacing/ICD lead leaving $\mathrm{l}$ inch $(2.5 \mathrm{~cm})$ exposed. The outermost electrode coil of the lead was then grasped with an artery clip and pulled out from the lead insulation. The coil was then cut at the external insulation, so the coil inside the insulation was partially unwrapped, leaving a space between the external

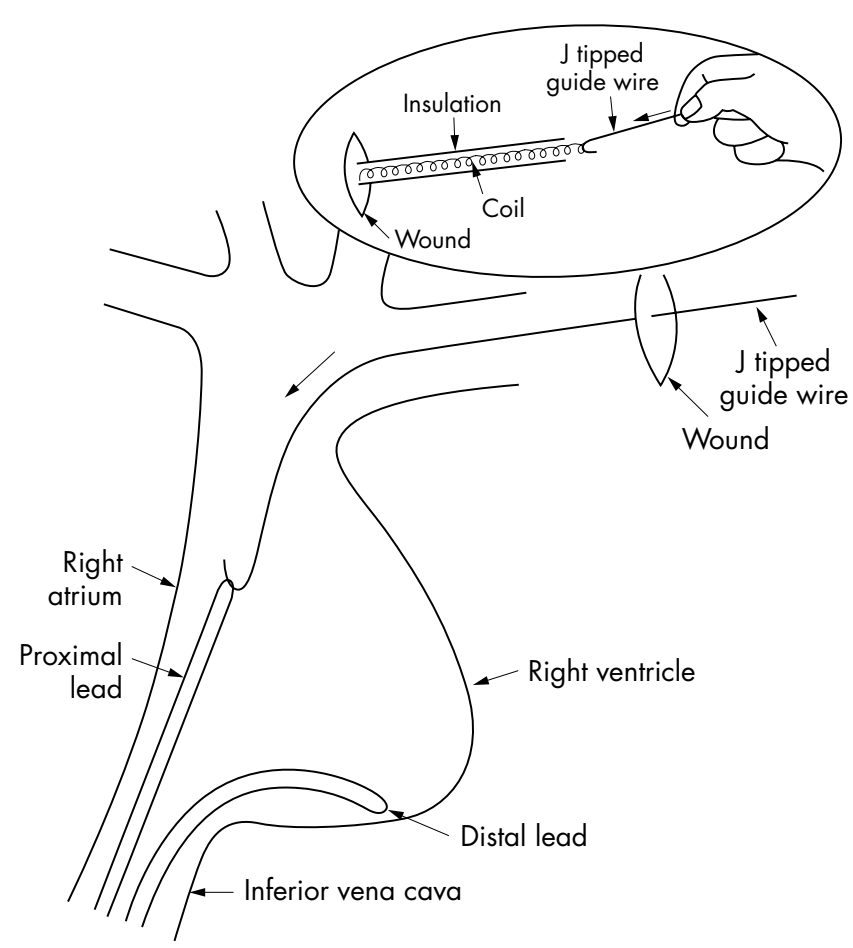

Figure 1 The generator has been removed and the lead to be extracted cut short. Retaining sutures and sleeves have been taken off the lead to be extracted. The coil has been grasped with artery clips and pulled out from the insulation. The coil is then cut at the point where it extends from the insulation, which leaves a gap between coil and insulation. A guide wire is pushed into the gap between the coil and the insulation so that it becomes firmly attached to the lead (inset). The guide wire has been pulled in behind the lead and is detached at the level of the right atrium.

insulation and the outer coil. A $140 \mathrm{~cm} 0.35$ inch J tipped guide wire was then introduced into the gap left between the coil and the insulation and pushed in until it was firmly attached (fig 1 inset). The proximal end of the lead was then pulled down into the right atrium from below while the guide wire was fed in behind. When the proximal lead/guide wire join reached the level of the right atrium the guide wire was fixed and the lead pulled so that the two separated (fig 1).

The lead was then extracted using previously described conventional counter-traction techniques. ${ }^{1}$ The guide wire was then used to introduce a $23 \mathrm{~cm}$ "peel-away" sheath (Daig Inc, Minnesota. USA) through which a replacement lead was then passed.

\section{Definitions}

Outcomes of the lead extraction were based on previously published recommendations. ${ }^{3}$ Complete success was defined 
Table 1 Lead extraction details

\begin{tabular}{|c|c|c|c|c|c|c|c|c|c|c|}
\hline Patient & Age & Indication & System & Extracted & Tech & Anaesth & Rem & Drag & Proc & Fluoro \\
\hline 1 & 50 & Failure & VVI & V & B & LA & $N$ & $1 / 1$ & 280 & 45.5 \\
\hline 2 & 51 & Pain & DDD & $V \times 2, A x 1$ & $S$ & LA & $N$ & $2 / 2$ & 130 & 104 \\
\hline 3 & 63 & Advisory & DDD & V & $S$ & LA & $N$ & $1 / 1$ & 135 & 35 \\
\hline 4 & 63 & Failure & DDD & $A, V$ & $\mathrm{~S}$ & LA & $N$ & $2 / 2$ & 175 & 20 \\
\hline $5^{*}$ & 41 & Failure & $\mathrm{AAI}$ & $A \times 1$ & $S$ & LA & $\mathrm{N}$ & $1 / 1$ & 300 & 58.8 \\
\hline 6 & 72 & Advisory & DDD & $\mathrm{V} \times 2, \mathrm{~A} \times 1$ & $S$ & LA & $\mathrm{N}$ & $2 / 2$ & 110 & 58.8 \\
\hline 7 & 73 & ICD & VVI & V & $S$ & GA & $\mathrm{N}$ & $1 / 1$ & 140 & 20 \\
\hline 8 & 60 & Failure & DDD & $A, V$ & $S$ & LA & Distal & $2 / 2$ & 150 & 34.2 \\
\hline 9* & 54 & Failure & DDD & $A, V$ & B & LA & $\mathrm{N}$ & $2 / 2$ & 90 & 10 \\
\hline 10 & 54 & Failure & VVI & V & $S$ & LA & $N$ & $1 / 1$ & 150 & 7.6 \\
\hline 11 * & 67 & Failure & DDD & V & B & LA & $\mathrm{N}$ & $2 / 2$ & 240 & 58.2 \\
\hline $12^{*}$ & 58 & $I C D$ & DDD & V & B & GA & $\mathrm{N}$ & $1 / 1$ & 60 & 12 \\
\hline 13 & 52 & Failed ICD & $I C D$ & $I C D$ & B & LA & $\mathrm{N}$ & $1 / 1$ & 100 & 28.4 \\
\hline 14 & 53 & Failed ICD & $I C D$ & $I C D$ & B & LA & Distal & $1 / 1$ & 120 & 75 \\
\hline Mean & 57.9 & & & & & & & & 155.7 & 40.5 \\
\hline SD & 9.0 & & & & & & & & 70.8 & 27.9 \\
\hline
\end{tabular}

*Patients with venous occlusion of the ipsilateral veins. Indication: Pain, painful pacemaker; Failure, lead failure and the patient is young ( $\leqslant 60$ years); Advisory, an advisory lead and the patient has requested lead extraction; ICD, patient required upgrading of pacemaker to ICD or replacement of a failed defibrillator lead. System: the system in situ requiring extraction; DDD, dual chamber; VVI and AAI, single chamber. Extracted: the number of each type of leads extracted. Tech: the system used to perform lead extraction; S, needle eye snare; B, Byrd femoral workstation. Anaesth: LA, local; GA, general anaesthetic. Rem: indicates whether any lead fragments were retained; N, none; distal, $<2 \mathrm{~cm}$ of distal lead was retained. Drag: how many leads were replaced by the drag through technique; ${ }^{*}{ }^{*}$ number of leads dragged through/number requiring extraction. Proc and Fluoro: procedure and fluoroscopy time (mins) including time for new implants.

as removal of all material from the vascular space. Partial success was defined as removal of all but a small portion of the lead. Clinical success was defined as achievement of all clinical goals associated with the indication for lead removal.

\section{RESULTS}

\section{Patients}

A total of 36 consecutive patients (22 male, mean (SD) age 63 (14) years) underwent extraction of 63 leads which had been in situ for 7.8 (4.6) years (7.3 (3.3) years for drag through patients). Nineteen patients had infected systems, one patient required radiotherapy for breast carcinoma on the ipsilateral side, one patient had a long section an active fixation lead floating free in the right atrium following a failed attempt to extract it from the superior approach at another centre, and one patient had a crush fracture between the clavicle and first rib. Therefore, 14 patients were suitable for lead extraction and immediate replacement of their system, four of whom required extraction of an old pacing/ICD system in order to implant an ICD. The indications for lead extraction in the remaining patients were lead failure in young patients $(\leqslant 60$ years) $(n=4)$, Telectronics Accufix lead removal requested by patient $(n=2)$, and venous occlusion $(n=4)$.

\section{Procedure}

Leads were extracted using a Byrd femoral workstation in six patients and a needle eye snare in eight patients (table 1). Clinical success was achieved in $100 \%$ of leads with $86 \%$ patients (91\% leads) having complete success. Partial success resulted from retention of the distal $2 \mathrm{~cm}$ of the lead ( $\mathrm{n}=2$ leads). Achieving venous access via the drag through technique was successful in all patients and was used to extract and replace 20 pacemaker/ICD leads. The drag through technique was successfully used in the eight patients with multiple leads. Two patients had three leads removed, including two previously abandoned ventricular leads, and six patients had one lead extracted only (five ventricular, one atrial) with functioning pre-existing leads left undisturbed. A single guide wire was dragged through and used to introduce two sheaths, allowing upgrade from a single chamber to a dual chamber pacemaker in one patient, otherwise individual guide wires were introduced for each lead being replaced. Two lead extraction procedures were performed under general anaesthetic (14.3\%) because of concurrent ICD implantation. The pacemaker lead drag through including reimplanta- tion procedure times were 155 (71) minutes and 41 (28) minutes. Mean stay in hospital was 1.5 (1.1) days.

\section{DISCUSSION}

Permanent pacemaker lead extraction has major complication rates of $1.4 \%^{1}$ and the results are similar using laser assisted lead extraction with major complication and mortality rates of 3.2\% and $0.6 \%{ }^{2}$ Although there is some theoretical advantage to femoral lead extraction, one limitation is that there is no vascular access for implantation of a new system. This is of particular relevance in those patients with subclavian or brachiocephalic vein occlusions, which occurs in up to $12 \%$ of patients with permanent pacemakers. ${ }^{4}$ Lead extraction and replacement of the lead on the same side as a vein occlusion avoids the possibility of bilateral subclavian occlusions, failure to cross a superior vena cava, or the need to implant a femoral system. Recent reports have demonstrated that replacing pacemaker leads through occluded veins is possible, particularly when using a laser assisted approach. ${ }^{5}$ This study has demonstrated that replacement of both permanent pacing and ICD leads is possible using the cheaper femoral approach, even when the veins are occluded. This was achieved without complication and was performed under local anaesthetic in most cases.

\section{Conclusion}

Cannulation of the veins used by non-functioning pacemaker/ ICD leads can be achieved using the drag through technique after lead extraction by the femoral approach. This allows introduction of new leads even through occluded veins.

Correspondence to: Dr Richard Schilling, St Bartholomew's Hospital, West Smithfield, London ECIA 7BE, UK;

richard.schilling@bartsandthelondon.nhs.uk

Accepted 24 October 2001

\section{REFERENCES}

1 Byrd CL, Wilkoff BL, Love CJ, et al. Intravascular extraction of problematic or infected permanent pacemaker leads 1994-1996. PACE 1999;22: 1348-57

2 Wilkoff $\mathrm{BL}$, Byrd $\mathrm{CL}$, Love $\mathrm{CJ}$, et al. Pacemaker lead extraction with the laser sheath: results of the pacing lead extraction with the excimer sheath (PLEXES) trial. J Am Coll Cardiol 1999;33:1671-6.

3 Love CJ, Wilkoff BL, Byrd CL, et al. Recommendations for extraction of chronically implanted transvenous pacing and defibrillator leads: indications, facilities, training. North American Society of Pacing and Electrophysiology Lead Extraction Conference Faculty. PACE 2000;23:544-51. 
4 Goto Y, Abe T, Sekine S, et al. Long-term thrombosis after transvenous permanent pacemaker implantation. Pacing Clin Electrophysiol 1998;21:1192-5.
5 Bracke FA, van Gelder LM, Sreeram N, et al. Exchange of pacing or defibrillator leads following laser sheath extraction of non-functional leads in patients with ipsilateral obstructed venous access. Heart 2000;83:e12.

\section{IMAGES IN CARDIOLOGY}

\section{Thoraco-omphalagus twins: heart to heart}

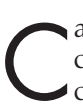

ardiovascular evaluation is a prerequisite before planning surgical separation of thoraco-omphalagus twins, as the degree of cardiac involvement is one of the limiting factors for success.

A set of 10 day old female thoraco-omphalagus twins presented with a protomesosystolic murmur suggesting a ventricular septal defect. Because of restrictive windows, cardiac ultrasound could not differentiate between a ventricular septal defect and a shunt between the left ventricle of one twin with the right ventricle of the other. Magnetic resonance imaging was not helpful but showed two separated hearts with a common pericardial sac. Cardiac catheterisation was performed through a retrograde approach-right femoral artery for the left twin and left femoral artery for the right twin. It showed that there was no communication between the hearts but a muscular septal defect in the right twin allowing filling of the pulmonary artery (arrow). Each heart has its own normal coronary artery supply. The apex of each heart enters the opposite chest. The anatomy was confirmed at surgery and the twins underwent successful separation.

\section{Beghetti \\ T Abdel-Massih \\ P Bonhoeffer \\ philipp.bonhoeffer@nck.ap-hop-paris.fr}

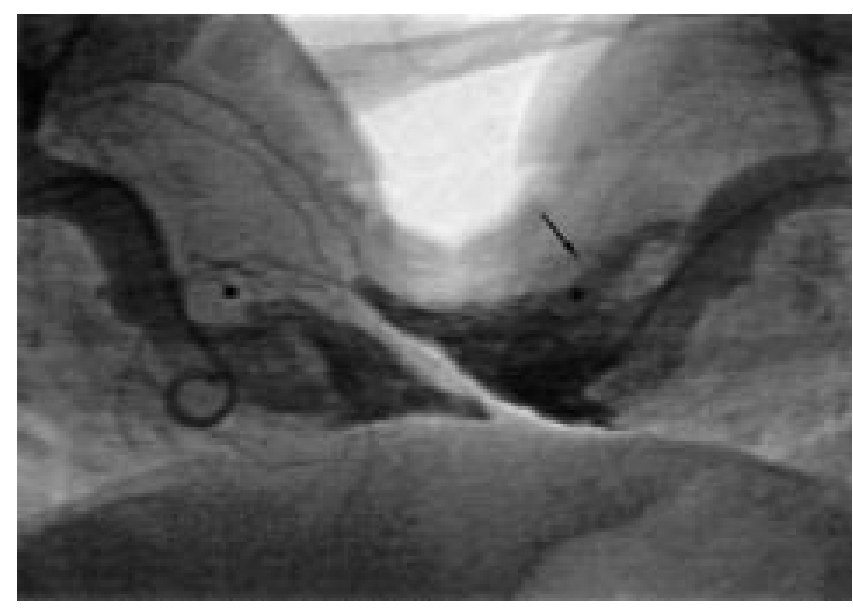

\section{Unroofed coronary sinus}

$A^{4}$ 47 year old woman was admitted to our hospital because of worsening shortness of breath on exertion which had initially occurred about 10 years previously. Transthoracic echocardiography showed the dilated coronary sinus protruding into the left atrium (below left, LA, left atrium; LV, left ventricle; Ao, aorta; arrow, dilated coronary sinus). The coronary sinus had a defect of $8 \mathrm{~mm} \times 18 \mathrm{~mm}$ in size through which a shunt flow from the left atrium to the sinus was demonstrated by colour Doppler echocardiography. Contrast (hand agitated saline) injected into the left

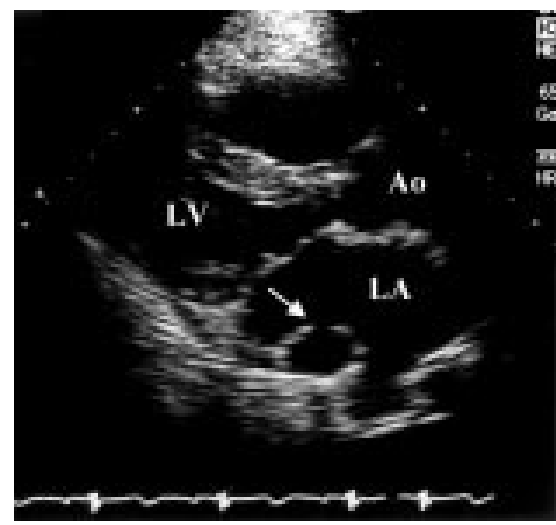

arm vein appeared in the coronary sinus before the right atrium. Estimated systolic pulmonary artery pressure from moderate tricuspid regurgitation was $42 \mathrm{~mm} \mathrm{Hg}$. By transoesophageal echocardiography with three dimensional reconstruction, we could clearly recognise the shape of the coronary sinus and the defect (below centre, viewed from the lateral side; right, viewed from the anterior side; arrow, coronary sinus with defect). From these findings, we diagnosed this patient as having an unroofed coronary sinus with persistent superior vena cava. Cardiac catheterisation

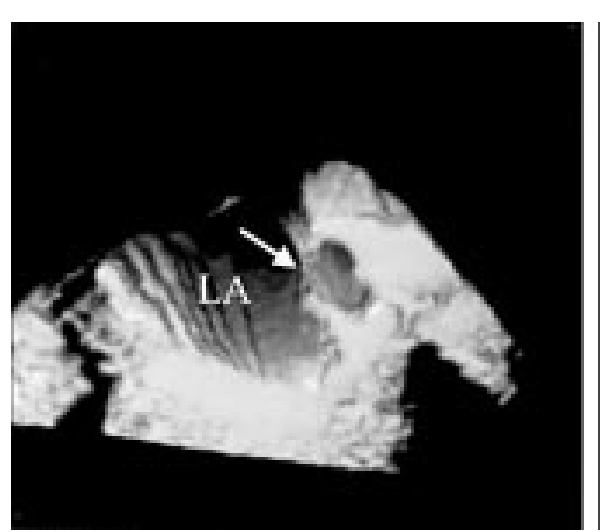

confirmed the diagnosis and demonstrated a significant left-to-right shunt with a shunt ratio of 2.53 . The patient was operated on and surgical findings were consistent with those revealed by echocardiography. The patient recovered uneventfully and was discharged 14 days later.

S Nakatani

K Katsuki

K Miyatake

nakatas@hsp.ncvc.go.jp

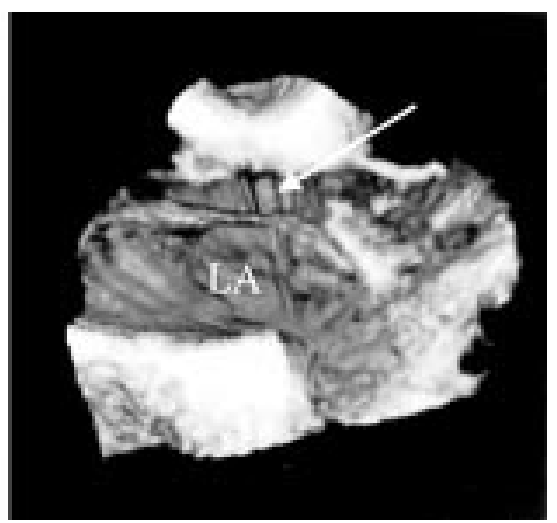

